\title{
LA CIENCIA COMO VIRTUD INTELECTUAL
}

1. Caracteres generales del hábito intelectual de la ciencia. 2. Teoría de la demostración: valor del silogismo. 3. Problemas de la inducción. 4. Jerarquia aristotélica de las ciencias. 5. Supuestos filosóficos de la doctrina aristotélica de la ciencia.

1. "Creemos poseer la ciencia de una cosa absolutamente, y no a la manera de los sofistas de modo accidental, cuando creemos conocer la causa por que dicha cosa es, y sabemos que esta causa es la de la cosa, y que no es posible que la cosa sea de otro modo de como es." 1

En estos términos define Aristóteles la ciencia; y si bien el pasaje pertenece a escritos suyos que no tienen que ver con la Etica, la ciencia ostenta allí también (por algo la Etica misma remite a los Analíticos para mayores especificaciones) su carácter típico de ser ante todo una $\tilde{E}_{\llcorner} \iota_{5}$ del sujeto cognoscente, un hábito intelectual, antes que un conjunto de proposiciones despersonalizadas. Es menester reparar en esto una vez más, ya que hoy estamos acostumbrados a ver la ciencia, al igual que los otros productos de la cultura, más bien como espíritu objetivado que como espíritu subjetivo. No fue ésta la perspectiva helénica, y por ello importa colocarnos en ella en el umbral mismo de cualquier elucidación o comentario.

La primera nota que sobresale en el conocimiento científico, con arreglo a la anterior definición, es la de ser un conocimiento por la causa. A reserva de explicar después el sentido lógico que este término puede tener aquí (tomando "causa" como el término medio del silogismo, merced al cual es posible la conclusión) parece cierto que Aristóteles ha entendido aquí "causa" también en el sentido ontológico que es habitual en su filosofía. Pero si así es ¿englobaría esta voz: airía, las cuatro bien conocidas causas aristotélicas, de suerte que por cualquiera de ellas pudiera obtenerse el conocimiento científico? La cuestión no es de fácil resolución, y más bien habría que inclinarse por la negativa en lo que concierne a la pareja de causas intrínsecas (material y formal), pues por ellas no sabemos apenas sino lo que una cosa es, o sea lo que expresamos en su definición; ahora bien, Aristóteles hace gran hincapié en que no es lo mismo definición y demostración (tipo por excelencia del conocimiento científico) y la definición, por tanto, no sería la ciencia sino cuando más incoativamente. En cambio, puede concederse sin dificultad que Aristóteles sí ha tenido presente, en el pasaje que comentamos, la otra pareja de causas extrínsecas (eficiente y final) como lo afirma Tricot a la zaga de

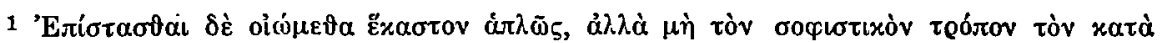

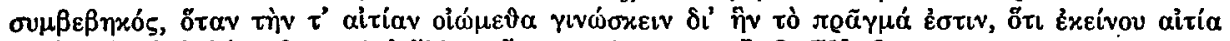

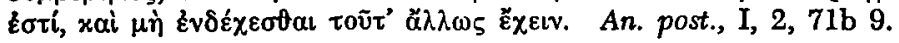


Trendelenburg, y ambas causas serían entonces, de acuerdo con esta interpretación, las que más valor tendrían en el conocimiento científico.

En contra de esta interpretación podrían aducirse, sin embargo, otros textos del Filósofo, como aquél en que nos dice ${ }^{2}$ que tenemos la ciencia de cada

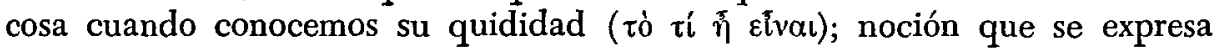
en la definición y puede alcanzarse no más que con el conocimiento de las causas intrínsecas: materia y forma, a las que corresponden respectivamente, en el orden lógico, el género y la diferencia. El problema tal vez pudiera resolverse, según lo que acaba de apuntarse, atribuyendo al conocimiento de la esencia (y consiguientemente a la definición) el carácter de ciencia, sólo que en estado incipiente, pues para su perfección sería necesario además el saber de las causas extrínsecas.

La ciencia debe ser además, en razón de su objeto, el conocimiento de

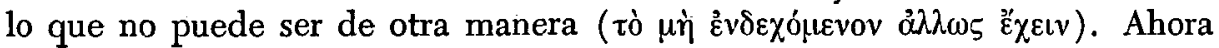
bien, una cosa puede ser de otra manera en razón del lugar o del tiempo, o de ambos predicamentos a la vez, como si una cosa cayera aquí y no en otro lugar, o ahora y no antes ni después, por lo cual la ciencia ha de ser, como dice Aristóteles, de lo universal y, ha de proceder por proposiciones necesarias, pues sólo lo necesario no puede ser de otra manera. ${ }^{3}$ A estas predicaciones añade aún Aristóteles las de ser el objeto de la ciencia, y en fuerza de los caracteres anteriores, inengendrable e incorruptible. ${ }^{4}$

De lo anterior se desprende que, hablando con todo rigor, apenas la metafísica y las matemáticas merecerían el dictado de ciencias con arreglo a los cánones aristotélicos. El Primer Motor que mueve todo el universo es, en

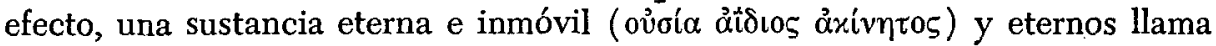
también Aristóteles a los objetos matemáticos. ${ }^{5}$ Lo que, en cambio, tiene que ver digamos con la actividad humana, sería más bien una $\pi 0 \alpha \gamma \mu \alpha \tau \varepsilon i ́ a$ que una $\dot{\varepsilon} \pi \iota \sigma \tau \dot{\eta} \mu \eta$, puesto que de tales cosas, en que todo parece ser variable, no puede haber "demostración", por más que Aristóteles parece conceder que a estas disciplinas podemos llamarlas ciencias por analogia. ${ }^{6}$ Su tratamiento, por ende, sería más bien propio de otro hábito intelectual, en concreto de la prudencia, como en su lugar veremos.

¿Qué habrá que decir entonces de la llamada por antonomasia ciencia natural, de la que ocupa un lugar intermedio entre los entes inmutables de la metafísica y la variabilidad esencialmente imprevisible de las acciones humanas, y cuyo objeto propio es determinar una legalidad, un orden constante en el acontecer extrahumano? La dificultad no podía pasar por alto a un in-

2 Met. VI, 6.

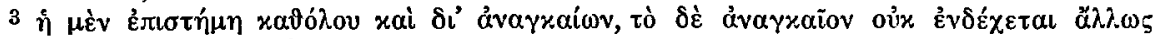

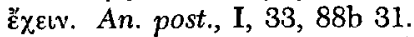

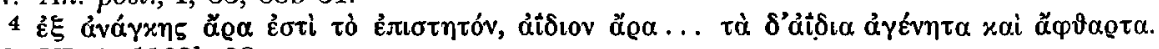

E. N., VI, 3, 1139b 28.

5 E. $N$., III, 3.

6 Cf. E. N., VI, 3 y 5. 
térprete tan avisado como Santo Tomás, y la resuelve diciéndonos que puede también haber ciencia aun de lo generable y corruptible, sólo que no será ciencia de los individuos particulares sujetos a la generación y corrupción, sino de sus "razones universales", las cuales sí son siempre y de necesidad.7 $\mathrm{Y}$ en otro lugar agrega que por más que las cosas sensibles sean corruptibles en particular, tienen con todo cierta eternidad si las consideramos universalmente. ${ }^{8}$

Alguna relación debe, pues, tener el objeto de conocimiento con lo universal para que de ello pueda haber ciencia. De lo particular como particular no puede tenerse ciencia, y este apotegma aristotélico continúa siendo válido hasta hoy, por lo menos en el consenso común de los filósofos. La escuela de Baden, como es bien sabido, realizó una labor por cierto meritoria para fundamentar el carácter científico de las llamadas ciencias idiográficas, en particular de la historia, mas para ello hubo de recurrir a la noción universal del valor, un universal in essendo, in valendo, in significando, como se quiera, pero que en todo caso constituye una unidad invariable de referencia con arreglo a la cual han de agruparse los hechos particulares en cuerpo de ciencia. Una pura descripción, en cambio, de hechos o cosas sin ningún criterio selectivo que permita reducirlos a una clase gobernada por una categoría unitaria e inmutable, sea ontológica o axiológica, no podría recibir el nombre de ciencia. Comprendemos bien, por lo demás, que el problema de las ciencias idiográficas no llegó a planteárselo formalmente Aristóteles, pero creemos con todo que están dentro de la noción aristotélica de ciencia $-y$ suponen su tácita admisión- estos intentos de fundar la legalidad científica de disciplinas que de algún modo se ocupan de lo particular bajo cierta razón universal.

Es conveniente añadir, antes de pasar adelante, que la proposición de que no hay ciencia de lo particular como particular ha de entenderse de lo particular que más corrientemente mentamos con esta expresión, es decir, de lo particular afectado por la materia, o sea de la sustancia corpórea. De otro modo, si tomásemos la susodicha proposición con absoluta generalidad, habría que negar desde luego el carácter de ciencia nada menos que a la metafísica, cuyo objeto primario es para Aristóteles no precisamente el ser en cuanto tal como algo común a todos los entes, sino el Ente único que es en sentido absoluto en sí y por sí, el Ens a se, Dios mismo, lo más individual e incomunicable que pueda imaginarse. Ahora bien, es claro que Aristóteles jamás pudo pensar en disputar siquiera la condición de ciencia a la que para él es precisamente l. ciencia por excelencia, la prima philosophia. Que ella tenga por

7 Potest autem etiam de generabilibus et corruptibilibus esse aliqua scientia, puta Naturalis; non tamen secundum particularia quae generationi et corruptioni subduntur, sed secundum rationes universales quae sunt ex necessitate et semper. Com. in Nic. Eth., VI, 3.

8 Etsi enim ista sensibilia corruptibilia sint in particulari, in universali tamen quandam sempiternitatem habent. In An. post., lib. I, c. 8, lect. 16. 
objeto a un Ente supremamente individual e incomunicable, en nada afecta al conocimiento absoluto que de él podamos tener (prescindiendo por ahora de nuestras limitaciones cognoscitivas) desde el momento que en él no difieren en nada la esencia y el individuo, la quididad y el supuesto, y no sólo no difieren, sino que hay en esas predicaciones identidad real absoluta. Lo mismo podría decirse (por más que en ellas hubiera cierta composición metafísica que no es del caso analizar) de toda otra sustancia espiritual en que la individuación no añade nada realmente a la quididad, sino que cada individuo constituye de por sí una especie, como lo sostuvo Santo Tomás al aplicar estos principios a los datos proporcionados por la Revelación sobre la existencia de los ángeles. Prescindiendo una vez más de lo deficiente que para nosotros pueda ser el conocimiento de esas sustancias, en razón de estar el entendimiento humano orientado ante todo a la quididad concreta en la materia sensible, el objeto mismo se ofrece de suyo con la misma limpidez y consistencia eidética que cualquier género o especie multiplicado en la sustancia corpórea y abstraído por nuestro entendimiento en el acto ideatorio.

En esta última, por el contrario, en la sustancia corpórea, la individuación no resulta de la forma, sino de la materia, y los atributos, por tanto, que definen al individuo en cuanto tal son atributos materiales, y por ende contingentes; y esto sin contar además con la otra contingencia de la circunscripción espacio-temporal en que forzosamente percibimos los individuos concretos. ¿Cómo podríamos tener ciencia, esta cognitio certa per causas, de lo que, en razón de los factores señalados, tan pronto es de este como del otro modo? Es verdad -y Aristóteles se hace expresamente cargo de la objeción ${ }^{9}-$ que en la percepción sensible se nos da de ordinario no sólo un hoc, sino un

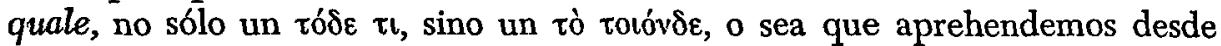
el primer instante de algún modo lo universal, pero con todo esto lo aprehendemos siempre en un lugar y momento determinados; ahora bien, lo universal es lo que es siempre y dondequiera ( Elval). Por estas razones, en conclusión, niega Aristóteles resueltamente, como ya lo había hecho Platón, que podamos adquirir la ciencia por la sensación. Estas son, en suma, las precisiones que era conveniente enunciar sobre la imposibilidad de tener conocimiento científico de lo singular como singular.

La apelación que hemos hechó a la metafísica, en prueba de los asertos anteriores, no la creemos fuera de lugar. Es preciso recordar que, como dice Maritain, ${ }^{10}$ para los antiguos era la dignidad eminente de la metafísica la que orientaba la noción misma de ciencia, su pauta y paradigma supremo, y no, como para los modernos, las ciencias de la naturaleza o cuando más las matemáticas. La ciencia por antonomasia, la metafísica, tenía por objeto directo la sustancia inmóvil, los entes positivamente ingenerables e incorruptibles, y

9 An. post., I, 31.

10 Les degrés du savoir, pág. 46. 
sólo por derivación como si dijéramos, en grado secundario; podían ser objeto de ciencia entidades negativamente incorruptibles como son las esencias o naturalezas inmutables de las cosas sensibles. En esta perspectiva histórica es menester situarse si se quiere alcanzar de todo esto una noción cabal.

Algo nos falta aún por declarar en el texto aristotélico con que iniciamos este capítulo, y es la precisión ulterior de que no basta conocer la causa para tener ciencia de un objeto, sino que es necesario además saber "que esta causa es la de la cosa". Hay que conocer, dicho en otros términos, el enlace preciso y necesario entre causa y efecto, pues de otro modo, como advierte Santo Tomás, no conoceríamos el efecto como real, en acto, sino a lo más como contenido virtualmente en la causa, lo cual no sería ciencia sino de manera accidental y bajo cierto respecto. ${ }^{11}$ Así, pues, el saber científico implica el conocimiento de la causa y del efecto, así como del nexo necesario e indefectible entre ambos tanto en el orden del ser como del conocer: Causae non tantum in cognoscendo et quoad nos, sed etiam in essendo.12

A la ciencia, y para hacer resaltar mejor su carácter, contrapone Aristóteles la opinión ( un conocimiento, es verdad, en que se ha dado un paso sobre la simple duda, estado de indecisión entre los extremos, pero que, con todo, no excluye en absoluto la posibilidad de error; y por esto los escolásticos definieron la opinión como la determinación del entendimiento hacia su objeto, pero siempre con temor de errar: Determinatio intellectus ad unum, cum errandi formidine. La opinión además puede darse tanto en razón de su objeto (lo particular y lo contingente) como porque el sujeto mismo no haya podido llegar aún por demostración a la aprehensión firme de un objeto de suyo universal y necesario, por lo cual admite Aristóteles ${ }^{13}$ que si bien no pueden darse en una misma persona simultáneamente ciencia y opinión con respecto a un mismo objeto, tal cosa es perfectamente posible en dos sujetos distintos. En cualquier hipótesis, no obstante, la opinión carece de esas notas que hacen de la ciencia un saber invariable e inconmovible, en reposo e inmóvil, predicados que una y otra vez, en el curso de sus escritos, le atribuye Aristó-

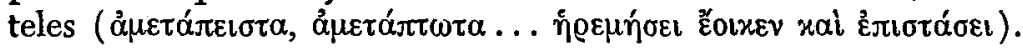

2. Preguntémonos en seguida cómo será posible alcanzar el saber científico de acuerdo con los caracteres que acabamos de asignarle.

Siendo la ciencia un saber por las causas, está dicho con ello que ha de ser un saber explicativo, y por lo mismo (al contrario de la intuición, como después veremos) un saber de evidencia mediata. Ahora bien, es asimismo

11 Si autem cognosceret causam tantum, nondum cognosceret effectum in actu, quod non est simpliciter, sed virtute tantum, quod est scire secundum quid et quasi per accidens. In An. post., ed. Vives, pág. 112.

12 Gredt, Elementa philosophiae aristotelico-thomisticae, Friburgo, 1921; I, pág. 165.

13 An. post., I, 33. 
obvio que un saber de este tipo no puede obtenerse sino por demostración, y por esto llama Aristóteles a la ciencia un hábito demostrativo ( $\varepsilon_{\xi} \varepsilon_{15} \alpha_{j} \pi 0 \delta \varepsilon ı$ $\tau(x \eta ́)$. Es, en otras palabras, la ciencia como virtud una fuerza intelectual merced a la cual alcanzamos por demostración un saber cierto de las cosas en sus razones universales y por sus causas.

Siendo ello así, el espíritu no puede seguir sino dos caminos en el proceso demostrativo: el primero de lo general a lo particular, la deducción, y el segundo a la inversa, de los casos particulares a la proposición universal, - sea la inducción. Este doble camino, que es desde entonces el abecé de la metodología científica, Aristóteles ha sido el primero en proponerlo con toda claridad y rigor, y sin ocultar sus preferencias por el procedimiento deducti-

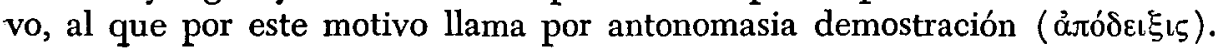
Y como esta demostración deductiva tiene su enunciación verbal en el silogismo, Aristóteles acaba diciendo que de nada podemos tener un saber fundado sino por silogismo o por inducción. ${ }^{14}$

No es de este lugar exponer la teoría del silogismo, y menos aún las reglas que en sus diversos modos y figuras tornan el silogismo válido. Baste indicar que el silogismo afirmativo se funda en el principio Dictum de omni, como el silogismo negativo a su vez en el otro principio correlativo Dictum de nullo, o en otras palabras, que lo que de manera universal y distributiva se afirma o niega de un sujeto, hay que afirmarlo o negarlo respectivamente de todos los individuos comprendidos bajo dicho sujeto. Ahora bien, esta subsunción o exclusión se realiza por la intervención del término medio, que por lo mismo ha de tomarse una vez por lo menos universalmente. Es así como merced a la comparación de los extremos con el medio, la conclusión dimana necesariamente, con la forzosidad que requiere la ciencia, de las premisas.

Todo esto es por supuesto de explorada lógica, y no ha de detenernos más. Lo que, en cambio, sí atañe a nuestro propósito, es la contribución real para la ciencia que respectivamente puedan representar, y en qué medida, tanto el silogismo como la inducción.

De la ciencia moderna, que opera sobre todo con base en la observación experimental, y cuyo método favorito es por ende la inducción, procede la ofensiva contra el valor demostrativo del silogismo aristotélico, entre cuyos antagonistas sobresale reconocidamente Stuart Mill. Según este filósofo, la conclusión del silogismo no demuestra nada, o por lo menos nada nuevo, desde el momento que la premisa mayor, para ser cierta, supone ya la conclusión y se apoya en la verdad de esta última. De este modo, si tomamos por ejemplo el socorrido silogismo en Barbara: "Todo hombre es mortal -Sócrates es hombre- Luego Sócrates es mortal", resultaría que la mayor no podría ser verdadera si no constase de antemano que Sócrates, Platón, Gorgias, etc. son mortales.

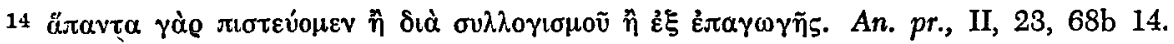


La objeción tiene indudable importancia en cuanto que apunta al carácter innegable que la ciencia ha de tener de conocimiento progresivo. Dejando de lado la cuestión de si los juicios analíticos pueden o no contribuir a la ciencia, lo cierto es que la mayor parte de los juicios que forman el acervo científico son, como lo vio Kant tan bien, juicios sintéticos, es decir, debe en ellos operarse un enlace de conceptos que no estén lógicamente contenidos uno dentro del otro. Si fuese cierto, por tanto, como lo pretende Mill, que la mayor del silogismo supone ya la conclusión, y que depende en su verdad de la verdad de esta última, es claro que no habríamos dado el menor paso adelante al formular la misma conclusión.

Las cosas, empero, no son tan sencillas como creyó verlas el filósofo inglés. Como buen empirista, Mill estaba en la firme persuasión de que todo juicio universal no es sino la suma de los juicios singulares obtenidos -esto último lo concedemos sin dificultad-por observación directa de los individuos, o en otros términos, que un juicio de esencia, universal y absoluto, como la mayor del silogismo en cuestión, no es sino la síntesis verbal de los juicios contingentes dados en la percepción sensible. Ahora bien, esto es precisamente lo que no habría admitido jamás la filosofía antigua, como tampoco la fenomenología moderna. La proposición de que todo hombre es mortal es un principio a priori, independiente de toda experiencia sensible, y cuya evidencia se percibe inmediatamente no del hechó de que veamos morir a Sócrates o a Platón, sino de la pura inspección a priori de la naturaleza humana, la cual, por estar compuesta de elementos contrarios, entre ellos el principio material, está ineludiblemente sujeta a la corrupción. ${ }^{15}$ Trátase aquí, como dicen los fenomenólogos, de una conexión esencial de validez apriorística, percibida en una intuición de esencia (Wesensschau) para la cual no hace falta en lo más mínimo haber procedido previamente a comparar entre sí los datos de una suma de experiencias singulares. Trátase, como decían por su parte los escolásticos, en este punto tan unánimes con la fenomenología, de la abstractio formalis, operación que se cumple, una vez más, no por la comparación de notas comunes percibidas en los individuos singulares, sino en la sola aprehensión de una esencia que se nos da como tal con sus notas y conexiones de necesidad intrínseca. No negamos por supuesto que la experiencia sea el camino propio para llegar a la noción de términos como hombre y mortal, como también, claro está, para su enlace fáctico; pero esto supuesto, bastaría de derecho la percepción de un solo hombre para que por la operación antes dicha (llámesela en términos escolásticos, fenomenológicos o como se quiera) pueda intuirse a priori la relación necesaria entre el predicado mortalidad y el sujeto hombre. De derecho decimos, porque bien que de ordinario formemos la mayor de aquel silogismo, como de tantos otros semejantes, con acopio mayor o menor de experiencias singulares, la premisa en cuestión no po-

15 Cf. Hugon, Cursus phil. thomisticae, I, pág. 175. 
dría tener la necesidad que ostenta si se apoyara tan sólo en esas experiencias y no pudiera mostrar a priori la conexión esencial entre sus términos.

Con lo anterior parece estar suficientemente demostrado que no hay ninguna necesidad, en estricto rigor, de que la premisa mayor suponga conocida la conclusión. En cuanto a la premisa menor, siempre en el ejemplo aludido, ella muestra el término que ha de ser sujeto de la conclusión subsumido bajo la especie universal de que se predicó antes la mortalidad, y. con este fundamento se le aplica el mismo predicado en la conclusión. Así pues, hay un progreso real en el conocimiento, por más que la conclusión esté contenida en las premisas como la ciencia en sus principios, como la planta en la semilla y como lo menos conocido y menos universal en lo que con mayor fulgor ostenta ambos atributos. Si así no fuese, no podría haber la concatenación necesaria entre los términos que supone la demostración, y las proposiciones científicas no serían sino proposiciones contingentes. Se podría por supuesto radicar el Apriori y la necesidad del enlace en el sujeto mismo del conocimiento, pero con esto entraríamos en una concepción distinta de la ciencia, cuyo estudio no nos incumbe aquí.

El silogismo tiene así, dentro de los presupuestos aristotélicos, un alto valor demostrativo, y es un instrumento eficaz para el progreso del conocimiento científico. Es posible que los ejemplos escolares con que aquí tenemos que operar no lo hagan ver así tan evidentemente, pero piénsese cómo proceden aun ciencias tan poco deductivas como pueden serlo, por ejemplo, la botánica o la zoología, las cuales disciernen estos o aquellos atributos a tales o cuales vivientes una vez que han podido subsumirlos dentro de la especie a la que, como resultado de investigaciones previas, pertenecen esas cualidades. ¿No es en verdad un procedimiento silogístico, de hecho si no de nombre? Por algo un espíritu tan universal como Leibniz no temía entorpecer, antes todo lo contrario, el progreso de las ciencias naturales al tributar al silogismo uno de los más encendidos elogios que sea posible hallar bajo la pluma de un filósofo. ${ }^{16}$

Aristóteles, está por demás decirlo, no parece haberse hecho cuestión expresa de las objeciones que andando el tiempo había de enderezarle en este capítulo Stuart Mill. De lo que sí se hizo cargo, y muy en pormenor, fue del modo precisó como el silogismo ha de dar razón de las causas cuyo conocimiento es, como estamos viendo, la arquitectura de la ciencia. En un pasaje fundamental de los Analíticos Posteriores, después de haber reafirmado su aseveración de que tenemos la ciencia cuando conocemos la causa, Aristóteles enumera una vez más las cuatro causas en este orden: la esencia o quididad (causa formal), la necesidad de que dadas ciertas cosas (causa material) se siga un efecto, el principio del movimiento (causa eficiente) y el fin en

16 Je tiens que linvention de la forme des syllogismes est une des plus belles de l'esprit humain et même des plus considérables. Nouv. ess., IV, 16. 
vista del cual tiene lugar ese efecto (causa final) y concluye diciendo que de todas estas causas hay demostración por el término medio. ${ }^{17}$

En la exégesis de Santo Tomás, a quien siguen Tricot y Maier, se interpreta la última frase en el sentido de que cualquiera de las cuatro causas puede servir de término medio en la demostración. ${ }^{18}$ De nuestra parte creemos también que éste fue el pensamiento del Filósofo, como se ve en los ejemplos de silogismo que aduce a continuación y que sumariamente pasamos a reproducir.

Para hacer ver cómo la causa material puede emplearse como término medio, Aristóteles formula el siguiente silogismo cuyo esquema, en la paráfrasis de Maier, ${ }^{19}$ es el siguiente:

Todo ángulo mitad de dos rectos (B) es un ángulo recto (A);

Todo ángulo inscrito en el semicírculo (C) es igual a la mitad de dos ángulos rectos (B); en consecuencia

Todo ángulo inscrito en el semicírculo (C) es igual a un ángulo recto (A).

Según lo explica el propio Aristóteles, B es la causa en virtud de la cual $\mathrm{A}$, ángulo recto, pertenece a $\mathrm{C}$, ángulo inscrito en el semicírculo; $\mathrm{o}$ en otros términos, $\mathrm{B}$ es el dato a partir del cual se sigue como consecuencia la pertenencia de $\mathrm{A}$ a $\mathrm{C}$. Y tiene aquí $\mathrm{B}$ razón de causa material, porque, según aclara por su parte Santo Tomás, cuando quiera que en matemáticas algo se demuestra del todo por las partes, la demostración es por la causa material, dado que, según dice Aristóteles en el libro II de la Física, las partes guardan con el todo una relación análoga a la de la materia con la forma.

Para mostrar el término medio como manifestativo de la causa formal, Aristóteles no recurre a otro ejemplo, sino que se limita a decir que, en el mismo silogismo citado, B se identifica con la quididad de A, puesto que es lo que la definición de A significa, como puede verse sin mayor esfuerzo con sólo que fijemos nuestra atención en la premisa mayor. Aristóteles remite además a otros lugares de los Analíticos Posteriores, ${ }^{20}$ donde ha mostrado cómo la atribución de un predicado esencial a un sujeto (su causa formal) en la conclusión, proviene necesariamente de haberse usado el mismo atributo como término medio, cosa que es fácil de comprobar por lo menos en el silogismo en Barbara.

De la causa eficiente empleada como término medio, Aristóteles pone

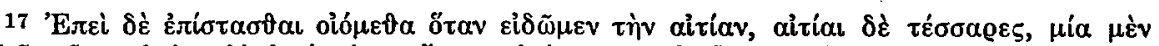

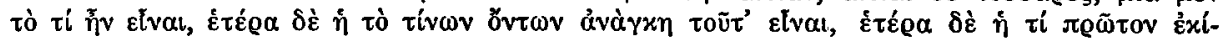

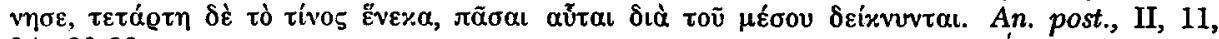
94a 20-23.

18 Quaelibet harum causarum potest accipi ut medium demonstrationis. In An. post., lib. II, lec. IX, y Maier, Die Syllogistik des Aristoteles, II, 2, pág. 227: "Die vier Ursachen lassen sich als Mittelbegriffe darstellen."

19 op. cit., II, 2, pág. 224.

20 Lib. II, caps. 8 y 10. 
como ejemplo un silogismo en que se haría ver cómo la agresión de los atenienses a Sardes, ciudad sujeta al rey de Persia, fue la causa determinante de las guerras médicas, según el siguiente esquema:

A quien ataca como agresor (B) se declara la guerra (A);

Los atenienses (C) atacaron como agresores (B); por tanto

A los atenienses (C) declararon (los persas) la guerra (A).

Sobre la causa final, por último, y en idéntica función silogística, Aristóteles aduce el caso de la respuesta que-daríamos a quien nos preguntara el fin último que perseguimos al estimular la digestión paseando después de cenar. La respuesta no podría ser otra sino que lo hacemos por conservar la. salud; de donde resulta el siguiente silogismo:

Para conservarme sano (B) debo tener buena digestión (A);

Salgo de paseo (C) para conservarme sano (B);

Salgo de paseo. (C) para tener buena digestión (A).

El ejemplo es artificioso, no hay duda, pues de ordinario solemos referirnos en último término al motivo final de nuestros actos, que en este caso es la salud, pero no pueden las cosas decirse de otro modo si la causa final ha de figurar como término medio y estar, por ende, ausente de la conclusión. Hay construcciones violentas del silogismo que, con todo, son legítimas, y la figura galénica no sería de ello el único ejemplo.

Por lo demás, nos damos bien cuenta de los reparos que por muchos motivos podrían hacerse a la doctrina y casuística que acabamos de resumir; por algo comentadores tan autorizados como Ross tienen estos pasajes de los Analíticos por particularmente arduos y oscuros. Sin poder por ahora entrar más a fondo en la cuestión, hemos creído indispensable mostrar, así sea esquemáticamente, cómo y por qué Aristoteles cree posible hacer del silogismo un instrumento eficaz, y por ventura el más excelente, del conocimiento científico. Sin detenernos en esto más, pasemos al otro procedimiento conducente al mismo fin, que es la inducción.

3. La inducción la define Aristóteles como el camino ascendente de lo singular a lo universal. ${ }^{21}$ Esta definición la traduce libremente Juan de Santo Tomás en esta forma: A singularibus sufficienter enumeratis ad universale progressio, ${ }^{22}$ donde la libertad, como se ve, consiste en introducir desde el principio la llamada inducción incompleta, cosa de la que por lo pronto no habla Aristóteles ni en el lugar citado ni en el capítulo de los Primeros Analíticos donde expresamente estudia la teoría de la inducción. ${ }^{23}$ Veamos pues en primer término la genuina doctrina aristotélica de la inducción completa, la que procede por la enumeración de todos los casos singulares (ท́ yò

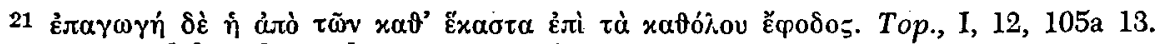
22 Cursus philosophicus thomisticus, I, pág. 60.

23 An. pr., II, 23. 


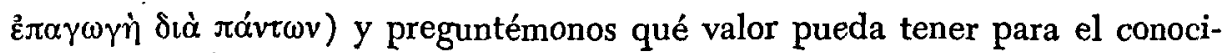
miento científico.

Lo primero que salta a la vista es que, como muy bien advierte Maier, ${ }^{24}$ lo que ante todo le interesa a Aristóteles es servirse de la inducción en orden al silogismo, o en otras palabras, como proceso de invención del término medio y la premisa mayor en el silogismo científico. Para comprobarlo así, la inducción misma puede reducirse, verbalmente por lo menos, a un silogismo, cuyo esquema, según lo encontramos en los tratadistas y de acuerdo con el ejemplo dado por el propio Aristóteles, podría ser el siguiente:

El hombre, el caballo y el mulo (B) viven largo tiempo (A);

El hombre, el caballo y el mulo (B) son todos los animales sin hiel (C); así pues

Todos los animales sin hiel (C) viven largo tiempo (A).

La conclusión es por supuesto inobjetable, dado que los términos de la menor son de hecho simplemente convertibles; pero si vemos las cosas bien, este llamado silogismo inductivo no tiene de tal sino la forma, pues desde luego el término medio -y es quizá la razón decisiva- no es un universal, sino una colección de singulares, y no puede por lo mismo reducirse, como quiere Lachelier, al modo Darapti de la tercera figura. ¿Para qué ha servido entonces? Pues sencillamente para encontrar el auténtico término medio y la premisa mayor del verdadero silogismo científico, que sería el siguiente en el modo Darii:

Todos los animales sin hiel (B) viven largo tiempo (A);

El hombre, el caballo y el mulo (C) son animales sin hiel (B);

El hombre, el caballo y el mulo (C) viven largo tiempo (A).

De acuerdo con el empirismo inglés, no habría ninguna diferencia esencial entre una y otra de las figuras lógicas que hemos trazado (el llamado silogismo inductivo y el silogismo demostrativo) por cuanto que la especie como tal: "todos los animales sin hiel", no sería sino la suma de los individuos singulares bajo ella agrupados. Este criterio, empero, lo hemos rechazado con antelación, y lo rechazan asimismo quienes, aun desde el punto de vista kantiano, concuerdan en este particular con Aristóteles, como Heinrich Maier, al decir: "El concepto general, que ocupa en el silogismo el lugar del término medio, es más que la mera suma de sus conceptos parciales, pues dispone además de una fuerza sintética merced a la cual está en capacidad de reunir las partes aisladas de su extensión en una unidad de validez real." ${ }^{25}$ Sea, pues, la esencia una entidad metafísica cum fundamento in re, sea un producto de la actividad sintética de la conciencia y sus formas categoriales, el universal que figura como término medio del silogismo demostrativo es cosa por entero diferente del universal fáctico del silogismo inductivo; es, como dice Maier, una unidad con validez real (realgiiltige Einheit) y las conexiones

24 op. cit., II, 1, pág. 370.

25 op. cit., II, 1, pág. 374. 
mostradas en la premisa donde aparece son conexiones de esencia esta vez, y no meramente generalización empírica de observaciones concretas. Con esto asume el silogismo demostrativo una dignidad incomparablemente mayor sobre el silogismo inductivo, pues lo que interesa a la ciencia es ante todo el hecho de que la falta de hiel en ciertos animales sea la causa necesaria de su larga vida, y no la comprobación fáctica de que tales o cuales animales viven largo tiempo, con la otra del mismo género de que todos esos animales carecen de hiel.

Son pues irreductibles uno al otro, digan lo que quieran ciertos lógicos, silogismo e inducción, ya que esta última no toma un término medio para hacer ver por su mediación la unión entre los extremos, sino que se limita a mostrar que un extremo o predicado conviene a un sujeto común porque conviene a los singulares o viceversa. Es simplemente un camino preparatorio de la auténtica dernostración científica cuando quiera que no podamos encontrar de otro modo las premisas del silogismo demostrativo.

Lo anterior no quiere decir sin embargo -y ésta es una nueva prueba de la heterogeneidad de ambos procedimientos- que la mayor del silogismo demostrativo, literalmente igual a la conclusión del silogismo inductivo en el ejemplo antes aducido, se apoye en esta última, es decir en la inducción, como en su razón formal, sino que, una vez establecida de cualquier modo que sea, así pueda ser con apoyo en múltiples experiencias, la proposición vale simplemente por la conexión esencial de los términos entre sí. Puede la experiencia hacerme ver mil veces que el todo es mayor que la parte, pero es claro que al enunciar este juicio en toda su generalidad no me apoyo en última instancia en ninguna experiencia, sino en la relación esencial entre el todo y las partes.

Se dirá que esta argumentación es pertinente en las ciencias matemáticas, pero no así en las ciencias naturales, en las cuales el juicio general dependerá siempre, en mayor o menor grado, de la experiencia. Supongamos que así sea (luego diremos sobre esto algo más) pero la única consecuencia que de ello habría que inferir, como lo hace Juan de Santo Tomás, sería la de que esas ciencias no son tan ciencias, en el sentido aristotélico del término, como otras en que basta ver una sola vez los términos para percibir ipso facto el enlace necesario entre ellos, como ocurre en metafísica y en matemáticas. ${ }^{26}$

Con todo ello, los escolásticos pensaron que aun esas ciencias de categoría inferior, como si dijéramos, no tienen una certeza meramente asertórica como la que correspondería a cualquiera de las llamadas verdades de hecho,

26 Unde si propositiones universales alicuius scientiae non sint ita abstractae et communes quod ex quocumque individuo manifestari possit ipsarum veritas, sed ex plurium numeratione et experientia pendeat, sicut scientiae naturales, non sunt ita certae sicut aliae scientiae abstractiores et communiores, ut Metaphysica et Mathematicae, quorum principia etiam in uno individuo habent totam certitudinem. op. cit. Marietti, I, pág. 200. 
sino que en virtud de otro principio que sería como el supuesto tácito de la inducción, podrían alcanzar una certeza, si no infalible, por lo menos válida para la generalidad de los casos. ¿Cuál es ese otro principio, ese alius loous arguendi, como dice Juan de Santo Tomás, que viene en auxilio de la inducción para robustecer lo más posible sus resultados? Pues es sencillamente, para decirlo en breve, el principio de que la naturaleza (es decir la esencia como principio de operaciones) actúa siempre uniformemente, a no ser que algo lo impida, y es la misma en todos los individuos singulares. Así lo expresa Hugon, reproduciendo la sentencia de Santo Tomás y los escolásticos: Natura determinatur ad unum, uno eodemque modo operatur nisi impediatur, eadem est in singulis. ${ }^{27}$ De este modo, mediante la percepción de un número suficiente de casos singulares $-\mathrm{y}$ tanto mejor si de todos- podemos llegar a la otra percepción, intelectual esta vez, de una esencia o naturaleza que a fuer de tal habrá de actuar siempre en el mismo sentido, y que siendo la misma así en los casos observados como en los no observados, nos garantiza, con respecto a estos últimos, la misma predicación que en lo tocante a los primeros. Quedará siempre, no obstante, un margen, que jamás podrá eliminarse del todo, de cierta inseguridad, en cuanto que puede haber causas (desde luego la intervención divina en el milagro) que pueden estorbar el curso ordinario de la naturaleza, al contrario de lo que pasa en matemáticas o metafísica, ciencias de inconmovible certeza.

El razonamiento que precede es, como se ve, absolutamente necesario en la fundamentación científica de la inducción incompleta, pero está implícito también (no obstante que los escolásticos pasen de ordinario por alto este punto) en la inducción per enumerationem simplicem, si es que, como hemos dicho, hemos de tener al cabo de ella la intuición de una naturaleza y no meramente una suma aritmética de experiencias singulares. Ahora bien, esto nos lleva a la elucidación del problema que dejamos antes pendiente, o sea el de saber si Aristóteles tuvo o no también en cuenta la inducción incompleta, o si por el contrario la escolástica fue en esto más allá del pensamiento del maestro.

Citar autores escolásticos en este punto podría quizá no ser muy convincente; pero aun entre los no escolásticos y entre los que más que nada son filólogos, hay quienes, como Ross, ${ }^{28}$ que se pronuncian resueltamente por el primer miembro de la alternativa. Fijémonos bien, nos dice Ross, y sin ir por lo pronto más lejos, en el mismo ejemplo aristotélico de la inducción completa. Las especies del género "animales sin hiel" están sin duda, en hipótesis, exhaustivamente enumeradas; pero ¿es que puede decirse otro tanto de los individuos comprendidos bajo dichas especies? ¿Es que por ventura han sido examinados todos los hombres, caballos y mulos para poder enunciar, como se hace en las premisas del silogismo inductivo, que todos ellos son

27 op. cit., I, pág. 423.

28 Aristotle, pág. 38 sigs. 
animales sin hiel y viven largo tiempo? ¿No estamos en estas premisas apoyándonos tácitamente, pero también inescapablemente, en una inducción incompleta? Y no sólo en este caso, sigue diciendo Ross, sino que en la generalidad de los ejemplos que encontramos en sus escritos (Ross puede afirmarlo así en mérito de su incomparable erudición) Aristóteles procede habitualmente de la especie al género, lo que supone forzosamente la legitimidad de la inducción incompleta en la formación de las proposiciones tocantes a la especie. Por otra parte, encontramos en los mismos escritos aristotélicos numerosos argumentos calificados de inductivos, en los cuales, sin embargo, la conclusión se apoya en pocos ejemplos cuando no en uno solo. ${ }^{29}$ Así pues, concluye Ross, no debemos ceñirnos muy estrechamente, al considerar la doctrina aristotélica de la inducción, al capítulo de los Analíticos Posteriores donde más largamente ha tratado de ella el Filósofo. "Esencialmente -dice el filólogo inglés- la inducción es para él un proceso no raciocinativo, sino de intuición directa, proceso mediatizado psicológicamente por una revista de casos particulares. ${ }^{30}$

Que la inducción aristotélica hay que entenderla pues en este sentido lato, lo confirmaría además aquel otro pasaje, que tanto trabajo ha dado a los intérpretes, donde Aristóteles dice que los principios de que procede el silogismo, no pudiendo a su vez ser obtenidos por silogismo, deben serlo por inducción, ${ }^{31}$ y en otro lugar paralelo parece decir que por inducción se adquiere incluso el conocimiento de los primeros principios. ${ }^{32}$ Ahora bien, si conforme a la primera norma de nuestra exegética hemos de interpretar a Aristóteles en forma que resulte congruente consigo mismo, no podemos creer que Aristóteles haya podido afirmar que a los primeros principios del conocimiento en sentido absoluto (principio de contradicción, principio de identidad, principio del tercero excluído) debamos llegar por inducción en la acepción rigurosa del término. Aceptar esta conclusión sería tanto como infirmar radicalmente la validez inconmovible de esos primeros principios, pues de otro modo no tendrían una evidencia apodíctica, y por otra parte se borraría toda distinción entre el hábito de la ciencia ( primeros principios (voṽs) hábitos indudablemente distintos, como lo comprobaremos en el siguiente capítulo. Si los primeros principios, en otras palabras, no se adquieren por evidencia inmediata, sino por demostración (y peor aún, si cabe, en forma inductiva) no habrá sencillamente primeros principios, pues cada demostración habrá de fundarse en otra, y así hasta el infinito, con lo cual jamás se cumpliría el absoluto imperativo aristotélico, válido tanto en el orden del ser como del conocer, de que en algún punto hay que detenernos.

29 Abundantes referencias en Ross, op. cit., pág. 39, n. 2.

30 op. cit., pág. 41.

31 E. N., VI, 3.

32 An. post., II, 19. 
¿Cuál podrá ser entonces una interpretación plausible de los citados pasajes? A nuestro modo de ver, no cabe sino una de las dos siguientes. La primera, que Aristóteles se esté refiriendo allí no a los primeros principios propiamente tales, sino a los que lo son para una ciencia determinada, como

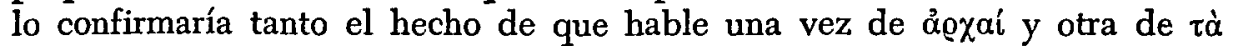

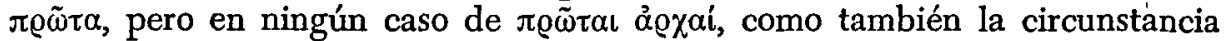
de que los ejemplos que trae en los Analíticos Posteriores de esos "principios" son en verdad principios genéricos para tal o cual ciencia (hombre, animal...) es decir para nociones o conceptos universales a cuya noticia sí es posible llegar por inducción.

La segunda interpretación sería, como lo cree Hessen, ${ }^{33}$ la de que Aristóteles está usando el término inducción, una vez más, en sentido muy amplio, por lo que Hessen estima que más bien convendría en este caso hablar no de inducción, sino de reducción para significar el tránsito de la percepción sensible a la intuición intelectual de los primeros principios. Posponemos el tratamiento de tan interesante sugestión para el capítulo siguiente, pues de momento sólo queríamos hacer ver el sentido lato, por no decir impropio, en que Aristóteles toma a menudo la inducción, como asimismo despejar la ilusión de que pueda ella ser el proceso conducente a la aprehensión de los

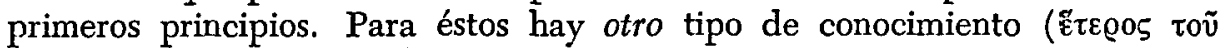

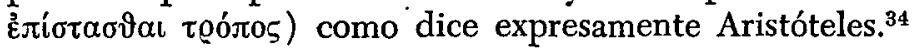

4. Aristóteles no ha procedido, como los modernos, a una verdadera clasificación de las ciencias, pero ha dejado los principios con arreglo a los cuales formuló la suya la escolástica medieval.

Los principios aristotélicos son bastante simples. Una ciencia es tanto más excelente que otra cuando da razón no sólo del qué (ỡı) sino del porqué $(\delta เ o ́ \tau \iota)$ es decir no sólo del hecho, sino de sus causas. ${ }^{35} \mathrm{~A}$ decir verdad, la primera no sería ciencia sino en estado embrionario, como quiera que la ciencia debe ser conocimiento por las causas. Y en segundo lugar, una cien-

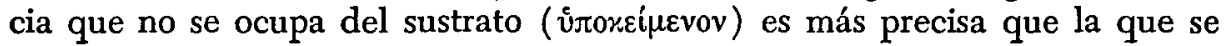
ocupa de él, como la Aritmética, dice Aristóteles, con respecto a la Harmónica. Ahora bien, por sustrato hay que entender aquí no el objeto mismo de conocimiento, sin el cual no habría ciencia posible, sino un objeto sensible

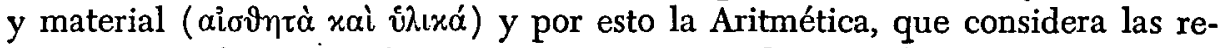
laciones numéricas en sí mismas, es superior a la Harmónica, que considera esas mismas relaciones en las cuerdas del instrumento musical. Por último, Aristóteles distingue aún entre la materia sensible ( inteligible ( duce que la ciencia absolutamente superior será la que se eleve aún por en-

33 Lehrburch der Philosophie, t. I: Wissenschafislehre; págs. 30 sigs.

34 An. post., I, 2.

$3 \overline{5}$ An. post., I, 27. 
cima de la materia inteligible y tenga sólo por objeto los puros inteligibles

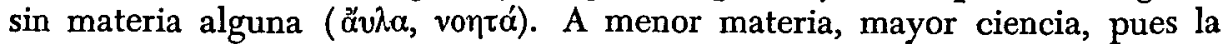
materia en sí misma, dice resueltamente Aristóteles, es incognoscible. ${ }^{36}$

Fundados pues en estos principios, los escolásticos dividieron las ciencias del ser real en física (ciencia natural) matemática y metafísica, con arreglo a los tres grados de abstracción de la materia que resultan de la doctrina anterior. El primer grado, fundatorio de la física, abstrae apenas de la materia sensible individual, pero conserva la materia sensible común, las notas constitutivas, por ejemplo, de una especie biologica. En el segundo grado, la matemática prescinde también de dicha materia y se queda apenas con la materia inteligible, esto es con relaciones numéricas que, por muy abstractas que sean, son siempre existencialmente un accidente de la materia sensible, bien que no tengamos ya en cuenta este carácter ${ }^{37}$ La metafísica por último, en el tercer grado de abstracción, se despoja de toda materia en absoluto y considera tan sólo los inteligibles puros del ser en cuanto ser.

Es bien evidente que dentro de la clasificación anterior no hay lugar para numerosas disciplinas que en la actualidad consideramos corrientemente como ciencias, o que por lo menos han hecho valer de tiempo atrás, con argumentos nada desdeñables, su pretensión al reconocimiento de esa dignidad. Serían, verbigracia, las llamadas ciencias morales y políticas, por lo menos en todo lo que no tenga relación con conceptos universales, y sería sobre todo, como ciencia-tipo de las ciencias idiográficas, la historia. Sobre esto, una vez más, es inútil hacerse ilusiones, pues Aristóteles es bien claro a este respecto. Para él, la historia no difiere de la poesía sino en que enuncia lo que ha sido, y no, como la poesía, lo que podría ser, y aún añade el Filósofo que la poesía es más filosófica y más seria ( @ov $)^{38}$ que la historia, por cuanto tiene que ver más que ésta con lo universal.

Así pues, no hay otro recurso que negar resueltamente que la ciencia deba serlo sólo de lo universal, o bien introducir de algún modo, de acuerdo con el espíritu aristotélico (aunque en todo caso contra la letra de los textos) lo universal en la historia, como lo sería por ejemplo la noción del valor, ya en la forma intentada por la escuela de Baden, ya como lo hacen ciertos neoescolásticos informados también en otras corrientes filosóficas contemporáneas. Para De Vries, por ejemplo, la categoría de la comprensión (Verstehen) que tiene su cabal aplicación en el conocimiento histórico, podría reducirse a la causa final ( $\sin$ la cual no comprenderíamos los hechos singulares) o dicho en términos modernos, al valor: "Pues la causa final es precisamente el bien, el valor que se presenta al espíritu y le incita a realizarlo y pone en movimiento las fuerzas eficientes." 39 Trátase, como se ve, de lucubraciones

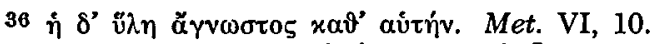

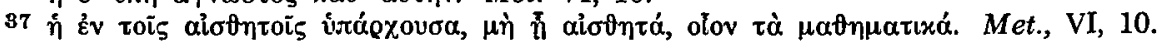

38 Poet., 9.

39 De Vries, Pensar y Ser, Buenos Aires, 1946; pág. 295. 
muy interesantes, y que acaso algún día permitan arrojar una conclusión cierta sobre el carácter científico de la historia. De momento, sin embargo, nos parece dudoso aún que, de acuerdo con los presupuestos aristotélicos, pueda ampliarse el cuadro de las ciencias más allá de lo que lo hicieron el mismo Aristóteles y sus comentadores medievales.

5. Nuestro propósito no es, sin embargo, el de examinar las limitaciones que muy bien puede tener la noción aristotélica de ciencia, para lo cual sería menester entrar en un análisis comparativo entre dicha noción y la que se ha tenido en los tiempos modernos, ni siquiera explorar más el contenido de las ciencias a que corresponde sin duda este carácter en la filosofía aristotélica. Estamos aquí tratando no de las ciencias, sino de la ciencia, y más precisamente aún, de la ciencia como hábito intelectual que permite, con relación a determinada esfera de objetos, alcanzar la verdad.

Mas por ello mismo, y a guisa de conclusión de este capítulo, no podríamos tampoco desentendernos de ciertos reparos que en el curso de la historia de la filosofía, y más acusadamente en la época moderna, se han hecho con respecto al núcleo mismo de la concepción aristotélica, y que de estar justificados, harían que no pudiera ésta sostenerse más. Es un problema que demandaría asimismo largo tratamiento, pero procuraremos ceñirnos brevemente a los puntos principales.

Hay, en primer lugar, objeciones que no afectan de suyo a la ciencia en general, sino sobre todo a las ciencias naturales, y no sólo en el sentido aristotélico del término, sino aun en su acepción moderna. La ciencia moderna, en efecto, no se preocupa de naturalezas o esencias, sino que cree estar constituída desde el momento que, con relación a determinado orden de fenómenos, puede establecer un conjunto sistemático de leyes, siendo ley, a su vez, la manera constante de verificarse un fenómeno. Cualesquiera que sean las diferencias entre la noción antigua y la moderna de ciencia, en esto convienen por lo menos, o sea en el carácter de necesidad que debe tener el conocimiento científico, y Kant lo defendió así con no menor vigor que Aristóteles. Aḥora bien, y prescindiendo de los argumentos del sensualismo antiguo y del empirismo inglés, argumentos suficientemente despachados en la historia de la filosofía, las teorías físicas modernas, particularmente en el campo de la física nuclear, han vuelto a poner en entredicho el cumplimiento fatal de las leyes naturales, o dicho más concretamente, de la ley de causalidad. Esta ley, en la época del positivismo, parecía tener tal soberanía que quería incluso reducir a ella la misma actividad humana; ahora en cambio se diría que los físicos quieren encontrar la libertad en el fondo mismo de la energía atómica. Si así fuere, es claro que las pretendidas leyes naturales no tendrían a lo más sino un valor estadístico. La inducción en especial sería no más que un cálculo de probabilidades, pues de que un agente en hipótesis libre se haya comportado hasta ahora de este modo, no se sigue que de repente no haya de com- 
portarse de otro. Podría hacerlo aunque nunca lo hubiese hecho, pues como dice el viejo adagio escolástico, a non esse ad non posse non valet illatio.

El problema, a decir verdad, no es precisamente nuevo. Con él ha tenido que habérselas la filosofía de la ciencia desde que los atomistas antiguos hablaron de aquel misterioso clinamen, al parecer indeterminado, en la caída de los átomos. En la imposibilidad de discutir ahora todos los aspectos de la cuestión, baste decir que mientras la física moderna no demuestre concluyentemente su punto de vista, podemos seguir ateniéndonos a principios que parecen estar hasta hoy suficientemente acreditados en filosofía. Del hecho de que a un fenómeno (y con mayor razón cuanto más difícil sea su observación directa) no podamos asignarle una determinación, no se sigue que sea en sí mismo indeterminado, sino que nuestros medios de control son insuficientes, y nada más. Por otra parte, no concebimos en el comportamiento de los entes sino estas dos categorías: determinismo y libertad, ya que un indeterminismo no libre iría directamente contra el principio de razón suficiente. Pero la libertad a su vez no la entendemos hasta hoy sino como propiedad de un ente espiritual, capaz de conocer las posibilidades que se abren ante su conducta. De consiguiente, y mientras no se demuestre la espiritualidad de la materia, de lo cual no vemos la menor señal, tendremos que continuar considerándola como sujeta al imperio inflexible en ese reino de la ley de causalidad. Por esta línea van más o menos los razonamientos de los neoescolásticos, a los que remitimos para una mayor exploración del problema. ${ }^{40}$

Más nos atañen aquí, sin embargo, las objeciones enderezadas con respecto al carácter mismo de los conceptos universales que constituyen la estructura de toda ciencia, aristotélica o no, que no pretenda ser ciencia de lo singular, así como la relación de dichos conceptos con la realidad. Toda ciencia es un conjunto de juicios -en esto hay también identidad absoluta entre Aristóteles y Kant- y todo juicio a su vez, según Aristóteles, es una síntesis de conceptos de tal modo que formen una unidad correspondiente a un ente o ser real. ${ }^{41}$ Ahora bien ¿'son esos conceptos un fiel traslado del ser real? Entre el vónua y tò ơ $v$ ¿hay adecuación y en qué grado? He aquí todo el problema de cuya solución depende el valor de la ciencia.

Es hasta hoy nuestra más sincera convicción, para ir derechamente al fondo del problema, que las impugnaciones que suelen hacerse a la teoría aristotélica de la ciencia en el punto neurálgico de la adecuación entre concepto y realidad, proceden en gran parte de no haber comprendido suficientemente lo que dicha adecuación quiere decir con toda precisión en el pensamiento aristotélico y de la escuela peripatética. Sólo así se explica que inclusive un autor como Hessen, por lo demás bien informado en esta filosofía, pueda decir que la concepción aristotélica de la ciencia es hoy insostenible por cuanto que el concepto pretende ser una copia (Abbild) de lo

40 Cf. De Vries, op. cit., págs. 275 sigs.

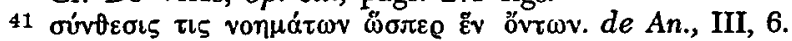


universal constitutivo de la realidad, cosas ambas que, en opinión del autor citado, son en absoluto inadmisibles. ${ }^{42}$

La dialéctica de Hessen es por démás brillante, pero es que jamás se tomaron literalmente expresiones como la anterior en la filosofía peripatética, por lo menos antes de su decadencia. Lo del concepto-copia es una mera invención, pues los escolásticos distinguieron siempre cuidadosamente entre el ser que las cosas tienen en la realidad (esse naturale) y el que tienen en nuestro entendimiento (esse intentionale); y de ahí que dijeran que el contenido del concepto universal, el universale directum, si bien se da realmente en las cosas mismas, no tiene en ellas la formalidad que adquiere luego en el acto ideatorio, y menos aún si del universal directo pasamos al universal reflejo, que es ya una elaboración de segundo grado, una reflexión, como su nombre lo indica, sobre el primer concepto, y cuyo resultado es la unidad, lógica que ubica dicho concepto en alguno de los cinco predicables. No era pues tan ingenua como se quiere hacerla aparecer esta noética del aristotelismo.

Mantuvieron estos filósofos, eso sí, con toda decisión, que nuestros conceptos tienen un fundamento in re y que lo que directamente percibimos son las cosas mismas, pues el concepto es aquello por lo que conocemos (id quo cognoscitur) y no aquello que primeramente conocemos (id quod cognoscitur) en la dirección natural del acto cognoscitivo y antes de reflexionar sobre su producto mismo, sobre el concepto. No por esto, sin embargo, pretendieron agotar intelectivamente la realidad, sino que tuvieron plena conciencia de que el conocimiento de lo singular como tal sólo es posible de modo indirecto, mediante lo que llamaban la conversión al fantasma imaginativo, y quedando siempre un residuo de ininteligibilidad, como lo dice el bien conocido adagio: Omne individuum ineffabile. En lo que discreparon tanto del nominalismo como del conceptualismo (bajo cuyas denominaciones incluimos respectivamente la filosofía de la vida y la filosofía crítica) fue en pensar que la realidad no es un puro caos de sensaciones, una corriente en eterno fluir e inasequible del todo a la razón, sino que hay en ella, pese a todos sus cambios y misterios, ciertas formas, contenidos, esencias, quididades o como quiera decirse, que el entendimiento puede desprender en la abtracción ideatoria y darles una formalidad propia en el concepto. Si el intelecto no tuviera este poder, no merecería este nombre, pues si se llama así es cabalmente porque, como dice Santo Tomás, puede leer dentro de las cosas e intuir su esencia: Dicitur autem intellectus ex eo quod intus legit, intuendo essentiam rei. ${ }^{43}$

Si los conceptos pues, y con ellos la ciencia, han de tener un valor, si no han de ser meros flatus vocis, habrá de atribuírseles cierto contenido, y si han de tener no sólo valor, sino valor real, habrán de corresponder, con todas las salvedades que se quiera, a la realidad de las cosas mismas. Lo que no ve-

42 op. cit., págs. 197 sigs.

43 In VI Eth., lect. 5. 
mos es que pueda haber algún medio entre el concepto como intelección de la realidad y el concepto como producto de la actividad sintética de la conciencia. El Erfassen que busca inútilmente Hessen como medio entre el $A b$ bilden y el Erzeugen, es, bien mirado, la aprehensión aristotélico-tomista de las formas inteligibles que fundamentalmente están en las cosas y formalmente, como tales, en nuestro entendimiento. Como en casi todo lo demás en que los campos se dividen entre los dos grandes príncipes de la filosofía, la cien: cia también, su esencia constitutiva, si ha de tener algún valor, ha de decidirse igualmente entre Aristóteles y Kant.

Lo que creemos en todo caso que permanecerá, cualesquiera que sean las reservas que puedan tenerse con respecto a la filosofía aristotélica de la ciencia, es el alto valor educativo y humano simplemente que alberga esta concepción de la ciencia como virtud intelectual. ${ }^{44}$ Para los griegos de la época prefilosófica la ciencia fue un don divino cuyos dispensadores eran Apolo y Palas Atenea, y Aristóteles es un heredero de esta estimación al colocar la ciencia, como lo hace, entre los grandes bienes de la vida, al lado de la amistad, el honor y otras excelencias del espíritu. ${ }^{45}$ Por esto hizo de ella ante todo, como hemos recalcado tantas veces, un hábito (jamás rutina, sino lo que quiere decir el sustantivo verbal de habere) un patrimonio, una posesión personal tan firme que por ello hubo de cargar el acento, antes que en la nuda posesión del dato científico, fácilmente sujeto al olvido, en la capacidad demostrativa del entendimiento humano, en su destreza para percibir las causas y las razones universales de las cosas. Y, por ello también, porque ambicionaba hacer estable la posesión de este bien, le fue forzoso inmovilizar hasta donde fuera posible el objeto del conocimiento científico, porque el sa-

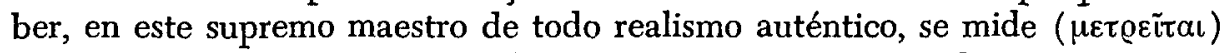
por el objeto, el scire por el scibile, a tal punto que no vacila en calificar de relación real, con toda la dependencia ontológica que esta categoría supone, la que hay del primero con respecto al segundo. ${ }^{46}$ En el orden del conocimiento dependemos totalmente del objeto y debemos entregarnos sin reservas a él dno es por vẹtura esta actitud de singular valor en la educación humana? Del objeto para nosotros, por el contrario, no hay sino una relación de razón. Poniendo una comparación que puede ser iluminadora, entre el scire y el scibile se establecen exactamente relaciones del mismo tipo que las que hay entre Dios y sus criaturas: reales las que nos ligan con El; de razón apenas las de Dios con nosotros. Devoción a la realidad, fidelidad al objeto, abstención de todo espíritu constructivista: éstos podrían ser los caracteres definitorios del valor educativo de la ciencia aristotélica.

En el espíritu que posee el saber, y como correlato o redundancia sentimental de esa relación de incomparable firmeza por ambos extremos, sobre-

44 Cf. Otto Willmann, Aristoteles der Erzieher, sobre todo cap. XI.

45 Ret., I, 6.

46 Top., I, 17; IV, 4. 
viene un estado de conciencia que Aristóteles describe reiteradamente con voces que significan reposo, serenidad y quietud; ${ }^{47}$ un estado, en suma, del mismo género del que corresponde a la posesión del bien y del objeto amado. ¿Hemos de culpar a Aristóteles porque no haya sido un pensador anacrónico, porque no haya sido un espíritu fáustico, porque haya preferido el gozo no mudable de la verdad poseída al afán incesante por perseguir un bien y una verdad que siempre nos escapan? Pero también ¿es que está demostrado, así sin más ni más, que esto y no aquello deba ser un valor más excelente y la condición del hombre?

Antonio Gómez Robledo

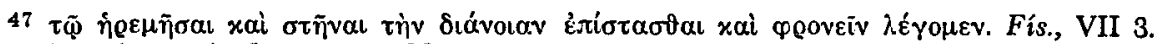

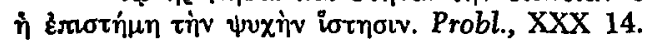

